\title{
Coronary Perforation to the Left Ventricular Cavity by a Guide Wire during Coronary Angioplasty
}

\author{
Makoto Tamura, M.D., Hirotaka Oda, M.D., \\ Tsutomu Mirda, M.D., Hironori Sato, M.D., \\ Norio Higuma, M.D., \\ and Tetsuro TOEDA, M.D.
}

\begin{abstract}
SUMMARY
We describe a case in which the guide wire penetrated through the left anterior descending artery into the left ventricular cavity during percutaneous transluminal coronary angioplasty. This complication of coronary angioplasty which had not been previously reported was suspected because of the characteristic motion of the guide wire on the $\mathrm{X}$-ray image and was confirmed by echocardiography. (Jpn Heart J 34: 633-637, 1993)
\end{abstract}

\section{Key Words:}

Iatrogenic coronary penetration Chronic total occlusion Percutaneous transluminal coronary angioplasty Echocardiography

\begin{abstract}
CORONARY perforation as a rare complication of percutaneous 1 transluminal coronary angioplasty is detected as extravasation or shunting to the cardiac chambers with injection of contrast medium. ${ }^{1-3)}$ In addition, there is one report of a case in which contrast echocardiography was found to be useful in determining the chamber into which an iatrogenic coronary fistula due to coronary perforation drained. ${ }^{4)}$ The purpose of this report is to describe a unique case in which coronary angioplasty was used to treat chronic total occlusion, and in which guide wire perforation through the left anterior descending artery into the left ventricular cavity was suspected because of the characteristic motion of the guide wire on the X-ray image and was confirmed by echocardiography.
\end{abstract}

\section{Case Report}

A 56-year-old male came to our hospital with the complaint of progressive chest pain. Two days before admission, he had suffered prolonged and severe chest pain. His electrocardiogram revealed $\mathrm{i} B \mathrm{BBB}$, abnormal $Q$ waves in leads

From the Department of Cardiology, Niigata City General Hospital, Shichikuyama, Niigata, Japan Address for correspondence: Hirotaka Oda, M.D., Department of Cardiology, Niigata City General Hospital, Shichikuyama 2-6-1, Nigata 950, Japan.

Received for publication December $9,1992$.

Accepted April 9, 1993. 
II, III and aVF, decreased height of $\mathrm{R}$ waves in $\mathrm{V}_{1-3}$ and $\mathrm{ST}$ depression with negative $\mathrm{T}$ waves in $\mathrm{V}_{4-6}$. Medical therapy relieved his anginal pain.

Coronary angiography performed ten days later revealed two occlusions, one in the mid left anterior descending artery and the other in the proximal right coronary artery. The distal left anterior descending artery was faintly collateralized by the diagonal artery and the first septal branch artery, and the distal right coronary artery was well collateralized by the left circumflex artery. Left ventriculography revealed severe hypokinesis of the inferior wall and hypokinesis of the anteroseptal wall. Thallium-201 scintigraphy demonstrated decreased initial distribution in the posterior-inferior and anteroseptal walls, and redistribution was noted in the anteroseptal wall. The electrocardiogram showed normal $\mathrm{R}$ waves in $\mathrm{V}_{1-3}$. The lesion responsible for the coronary event was considered to be located in the left anterior descending artery.

Coronary angioplasty was performed for the lesion of the left anterior descending artery about 2 months after the acute coronary event because of evi-

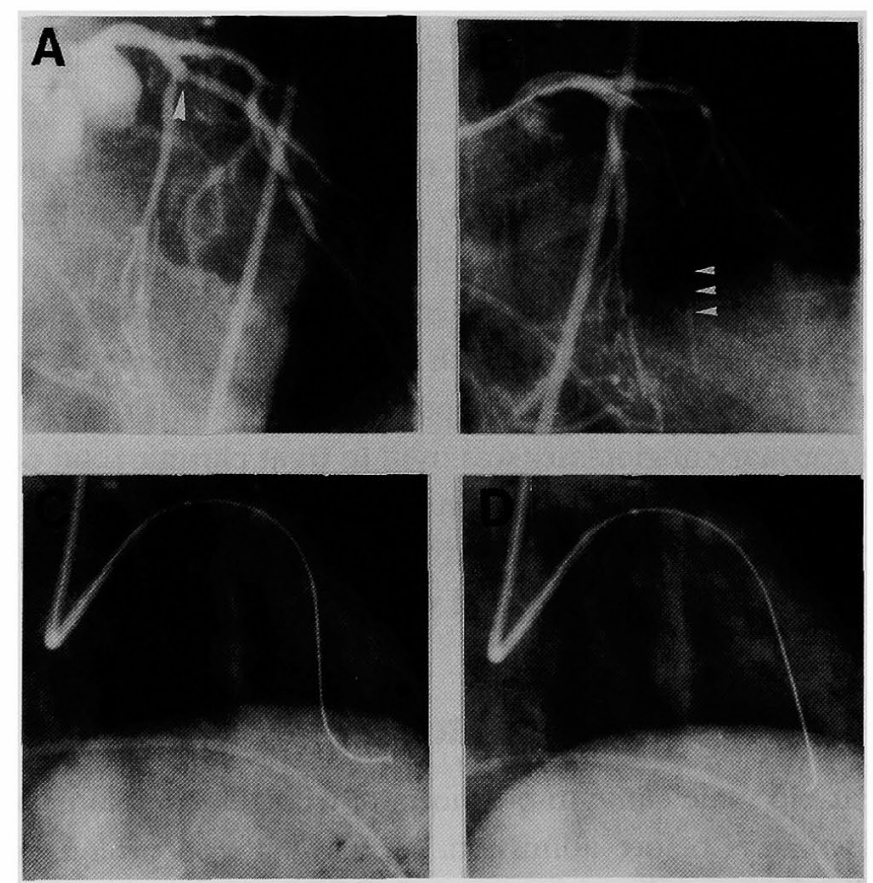

Fig. 1. Penetration by guide wire during coronary angioplasty for occluded left anterior descending artery lesion. A: the eccentric and tapering lesion in the left anterior descending artery in $30^{\circ}$ left anterior oblique- $30^{\circ}$ cranial view. B: position of advanced guide wire and contrasted left anterior descending artery in $15^{\circ}$ left anterior oblique $-30^{\circ}$ cranial view. $\mathrm{C}$ and $\mathrm{D}$ : swinging of the guide wire in the left ventricular cavity. Large arrow indicates the tapering lesion of the occluded left anterior descending artery. Small arrows indicate left anterior descending artery filled by collaterals. 
dence of ischemia in the area supplied by the occluded left anterior descending artery. Coronary angioplasty was performed with an 8F Judkins L-4 guiding catheter (Angiomedics), high-torque floppy 0.018 guide wire (ACS), and a Pinkerton 2-mm balloon (ACS). As the guide wire could not be made to cross the lesion, which showed tapering and eccentric morphology with an occluded length of about $2 \mathrm{~cm}$ (Fig. 1A), we advanced the balloon close to the occlusion and replaced the wire with a stiffer wire (USCI standard 0.016 guide wire). The tip of the wire passed the tapering lesion and progressed toward the septum. We assumed that the guide wire had entered into the septal branch after passing the thrombus or the atheroma of the totally occluded lesion. We attempted to advance the guide wire toward the left anterior descending artery guided by visualization of the distal arterial segment in left and right anterior oblique views by contrast medium injections. After we manipulated the wire, the tip of which was in the occluded lesion, with slight resistance, the guide wire progressed easily toward the left anterior descending artery. The advancing wire seemed to be in the distal segment of the left anterior descending artery filled by ipsilateral collaterals (Fig. 1B).

When we advanced the guide wire further, we saw that the tip of the wire suddenly began to swing in rhythm with the heart beat like a whip (Fig. 1CD). Careful radioscopic examination in several views revealed that the tip of the wire was not in the left anterior descending artery or in the pericardial space because it swung very widely. Echocardiography clearly revealed the high density linear echo of the wire in the left ventricular cavity from the septal wall to the posterior wall (Fig. 2); the linear echo disappeared when the guide wire was withdrawn. After the withdrawal of the guide wire, coronary angiography demonstrated no

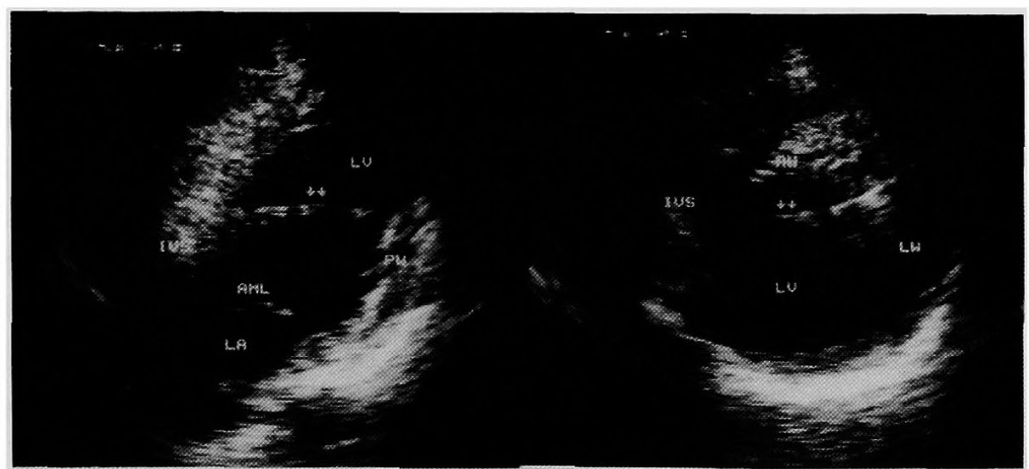

A

B

Fig. 2. Two-dimensional echocardiogram obtained by two-chamber view (A) and short-axis view (B). Arrows indicate the guide wire in the left ventricular cavity. $\mathrm{AML}=$ anterior mitral leaflet; $\mathrm{AW}=$ anterior wall; IVS=interventricular septum; $\mathrm{LA}=\mathrm{left}$ atrium; $\mathrm{LV}=$ left ventricle; $\mathrm{LW}=$ lateral wall; $\mathrm{PW}=$ posterior wall. 
leakage of contrast medium into the left ventricular or pericardial space, and echocardiography showed no evidence of pericardial fluid. During this time, the patient had no symptoms and there was no change in hemodynamic status.

\section{Discussion}

During coronary angioplasty, coronary perforation can be caused by guide wire penetration or by coronary rupture due to balloon dilation in the subintima or oversized dilatation of coronary segments. In reported cases of coronary angioplasty with sequela of an iatrogenic coronary artery fistula to the cardiac chambers, the fistula developed after balloon inflation without inappropriate tracking of the guide wire. ${ }^{1-3)} \mathrm{In}$ addition, there are some reports of guide wire perforation through the coronary arteries into the pericardial space, ${ }^{5)}$ although we are not aware of any rcport of wire perforation into the left ventricular cavity such as in our case.

However, it was not clear which part of the coronary artery was perforated. The left ventricular wall is so thick that it is not likely that the guide wire would go through the entire myocardium. Estes et $\mathbf{a}^{\left.{ }^{b}\right)}$ reported that the epicardial branches of coronary arteries send deep tributaries at right angles into the myocardium, and that these tributaries are 400 to 1,500 micra in diameter; they quickly divide into class $A$ and class $B$ vessels. The class $B$ vessels extend to the inner layers of the myocardium with almost no reduction in size. In our case, the guide wire progressed toward the septal wall without strong resistance and we believe that, since its diameter was about 400 micra, it could enter into the septal artery as well as into a "class B" artery and proceed to the subendocardial layer to penetrate into the left ventricular cavity.

When coronary angioplasty is used for chronic total occlusions, contrast medium does not always enter the branches in the lesion and it is therefore not clearly visible. If the guide wire proceeds only toward such arteries, it is difficult to perform coronary angioplasty successfully, and the coronary artery may be perforated, as occurred in this patient. In the event of perforation, detection of wire mal-tracking before balloon inflation can prevent the serious complications of coronary fistula to the left ventricular cavity or increased ischemia. During manipulation of the wire, it is important to verify not only wire tracking with injection of contrast medium but also the motion of the wire; the tip of a wire which is attached to the left ventricular endocardium will move like a whip, as in our case. We emphasize that echocardiography was very useful in confirming the presence of the wire in the left ventricular cavity. 


\section{References}

1. Meng RL, Harlan JL: Left anterior descending coronary artery-right ventricle fistula complicating percutaneous transluminal angioplasty. J 'Ihorac Cardiovasc Surg 90: 387, 1985

2. Cherry S, Vandormael M: Rupture of a coronary artery and hemorrhage into the ventricular cavity during coronary angioplasty. Am Heart J 113: 386, 1987

3. Iannone LA, Iannone DP: Iatrogenic coronary artery fistula-to-left ventricle following PTCA; a previously unreported complication with nonsurgical management. Am Heart J 120: 1215, 1990

4. Grill HP, Chew PH, Weiss JL, Merillat JC, Hill G, Cadden J, Brinker JA: Contrast echocardiographic diagnosis of PTCA-induced coronary artery-left ventricle fistula. Am Heart J 121: 194, 1991

5. Meier B: Benign coronary perforation during percutaneous transluminal coronary angioplasty. $\mathrm{Br}$ Heart J 54: 33, 1985

6. Estes EH, Entman ML, Dixon HB, Hackel DB: The vascular supply of the left ventricular wall. Am Heart J 71: 58, 1966 